\title{
QUOTIENTS OF COMPLETELY REGULAR SPACES
}

\section{J. HIMMELBERG ${ }^{1}$}

In a previous paper [1] we gave necessary and sufficient conditions for a quotient space of a pseudo-metrizable space to be pseudometrizable. In this note we give a short proof of the corresponding theorem for preservation of complete regularity by quotient maps. The proof specializes in an obvious way to the pseudo-metric case and has the advantage that, unlike the proof in [1], it requires neither the use of uniformities nor the complicated construction of that paper. Moreover, we obtain an interesting explicit definition of a pseudo-metric (or, in the complete regularity case, a defining family of pseudo-metrics) for the quotient space.

For the most part the terminology here is standard. But we wish to make some things explicit. If $p$ is a pseudo-metric for $X$, and if $\epsilon>0, x \in X$, and $A, B \subset X$, then

$$
\begin{aligned}
N_{\epsilon}[x] & =N_{p, \epsilon}[x]=\{z \in X \mid p(z, x)<\epsilon\}, \\
p(A, B) & =\inf \{p(a, b) \mid a \in A, b \in B\}, \\
N_{\epsilon}[A] & =N_{p, \epsilon}[A]=\{z \in X \mid p(z, A)<\epsilon\} .
\end{aligned}
$$

The topology on a space $X$ defined by a family $P$ of pseudo-metrics for $X$ is the topology with $\left\{N_{p, \epsilon}[x] \mid p \in P, \epsilon>0, x \in X\right\}$ as subbase. (We do not require in the above definition that $P$ separate points; so the topology generated by $P$ need not be Hausdorff.) Recall that a topology on $X$ is completely regular if and only if it can be defined by a family of pseudo-metrics.

THEOREM 1. Let $f$ be a function from a completely regular space $X$ onto a topological space $Y$, and suppose that $Y$ has the quotient topology relative to $f$. Then the following assertions are equivalent:

(1) $Y$ is completely regular.

(2) There exists a family $P_{0}$ of pseudo-metrics defining the topology of $X$ and a subbase $S$ of the topology of $Y$ such that for each $G \in S$ there exists $p \in P_{0}$ and a set $\{\epsilon(y, p) \mid y \in G\}$ of positive real numbers satisfying

(i) $N_{p, \epsilon(y, p)}\left[f^{-1}[y]\right] \subset f^{-1}[G]$, if $y \in G$,

(ii) $p\left(f^{-1}[y], f^{-1}[z]\right) \geqq \epsilon(y, p)-\epsilon(z, p)$, if $y, z \in G$.

(3) There exists a family $P_{0}$ of pseudo-metrics defining the topology of $X$ such that the topology of $Y$ is defined by the family $Q=\left\{q_{p} \mid p \in P_{0}\right\}$

Received by the editors March 20, 1967.

1 This research was supported in part by the National Science Foundation (GP$6490)$. 
of pseudo-metrics defined by

$$
q_{p}(y, z)=\inf \sum_{i=1}^{n} p\left(f^{-1}\left[y_{i-1}\right], f^{-1}\left[y_{i}\right]\right),
$$

where $y, z \in Y, y_{i} \in Y$ for all $1 \leqq i \leqq n$, and the infimum is taken over all finite chains $y=y_{0}, y_{1}, \cdots, y_{n}=z$.

Remark. Assertion (1) implies the existence of a single family $P_{0}$ which satisfies the requirements of both (2) and (3). Also the proof of $(1) \Rightarrow(2)$ requires only that $f$ be continuous, and not necessarily that $f$ also be a quotient map.

Proof of $(1) \Rightarrow(2)$. Let $Y$ be completely regular, let $P, Q$ be families of pseudo-metrics which define the topologies of $X, Y$, respectively. For each $q \in Q$, let $\delta_{q}$ be the topology on $Y$ defined by $q$. Then let $s=\bigcup\left\{s_{q} \mid q \in Q\right\}$. For each $(p, q) \in P \times Q$, define $p_{q}: X \times X \rightarrow R$ by

$$
p_{q}(x, y)=p(x, y)+q(f(x), f(y)), \quad \text { if } x, y \in X .
$$

Let $P_{0}=\left\{p_{q} \mid(p, q) \in P \times Q\right\}$. Trivially, each member of $P_{0}$ is a continuous pseudo-metric for $X$; so the topology on $X$ defined by $P_{0}$ is smaller than the topology defined by $P$. Thus, since $p_{q}(x, y) \geqq p(x, y)$, for $(p, q) \in P \times Q$, and $x, y \in X$, it follows that $P_{0}$ and $P$ define the same topology, i.e., $P_{0}$ defines the given topology on $X$. Now let $G \in \mathcal{S}$, say $G \in S_{q}$, with $q \in Q$, and let $p \in P$ be arbitrary. Define

$$
\epsilon\left(y, p_{q}\right)=q(y, Y-G), \quad \text { if } y \in G .
$$

By the way $q$ was chosen, it is trivial that each such $\epsilon\left(y, p_{q}\right)$ is positive. It is also easy to check (i) and (ii) of (2).

Proof of $(2) \Rightarrow(3)$. Let $P_{0}$ and $\delta$ be given as in (2) and let $Q$ be defined as in (3). It is easily shown that each $q_{p} \in Q$ is a pseudo-metric for $Y$. Moreover for each $p \in P_{0}, f$ is continuous (in fact decreases distances) if $X, Y$ are given the topologies defined by $p, q_{p}$, respectively. Thus $f$ is continuous relative to the topologies defined by $P_{0}$ and $Q$. All that remains to be shown is that the topology defined by $Q$ is larger than the quotient topology on $Y$. To do this it is sufficient to show that each member of $S$ is open in the topology defined by $Q$. So let $G \in S$, and let $p \in P_{0}$ and $\{\epsilon(y, p) \mid y \in G\}$ be as given by (2). Then we claim that, for all $y \in G, q_{p}(z, y)<\epsilon(y, p) \Rightarrow z \in G$.

For suppose $q_{p}(z, y)<\epsilon(y, p)$. Then there exists a chain $y=y_{0}, y_{1}$, $\cdots, y_{n}=z$ of points of $Y$ such that

$$
\sum_{i=1}^{n} p\left(f^{-1}\left[y_{i-1}\right], f^{-1}\left[y_{i}\right]\right)<\epsilon(y, p) .
$$


In particular, $p\left(f^{-1}[y], f^{-1}\left[y_{1}\right]\right)<\epsilon(y, p)$. This means that $p\left(f^{-1}[y], u\right)$ $<\epsilon(y, p)$ for some $u \in f^{-1}\left[y_{1}\right]$. Consequently, $u \in f^{-1}[G]$ and $y_{1}=f(u)$ $\in G$.

Now apply (ii) of $(2)$ to $\left({ }^{*}\right)$ to obtain

$$
\begin{aligned}
\sum_{i=2}^{n} p\left(f^{-1}\left[y_{i-1}\right], f^{-1}\left[y_{i}\right]\right) & <\epsilon(y, p)-p\left(f^{-1}[y], f^{-1}\left[y_{1}\right]\right) \\
& \leqq \epsilon(y, p)-\epsilon(y, p)+\epsilon\left(y_{1}, p\right) \\
& =\epsilon\left(y_{1}, p\right) .
\end{aligned}
$$

Thus by repeating the argument following the inequality $\left({ }^{*}\right)$, we deduce successively that $y_{1}, y_{2}, \cdots, y_{n}=z$ all belong to $G$. We have thus proved that $G$ is open relative to $q_{p}$, and hence is open in the topology defined by $Q$.

ProOF of $(3) \Rightarrow(1)$. Trivial.

A simplified $\left(P_{0}\right.$ and $Q$ in the statements of (2) and (3), and $P, Q$ in the proof of $(1) \Rightarrow(2)$ will all have only one element) version of the above argument now gives the following pseudo-metric version of Theorem 1.

Theorem 2. Let $f$ be a function from a pseudo-metrizable space $X$ onto a topological space $Y$, and suppose that $Y$ has the quotient topology relative to $f$. Then the following assertions are equivalent:

(1) $Y$ is pseudo-metrizable.

(2) There exists a pseudo-metric $p$ defining the topology of $X$ and a subbase $\&$ for the topology of $Y$ such that for each $G \in \mathcal{S}$ there exists a set $\{\epsilon(y) \mid y \in G\}$ of positive real numbers satisfying

(i) $N_{\epsilon(y)}\left[f^{-1}[y]\right] \subset f^{-1}[G]$, if $y \in G$,

(ii) $p\left(f^{-1}[y], f^{-1}[z]\right) \geqq \epsilon(y)-\epsilon(z)$, if $y, z \in G$.

(3) There exists a pseudo-metric $p$ defining the topology of $X$ such that the topology of $Y$ is defined by the pseudo-metric $q$ defined by

$$
q(y, z)=\inf \sum_{i=1}^{n} p\left(f^{-1}\left[y_{i-1}\right], f^{-1}\left[y_{i}\right]\right),
$$

where $y, z \in Y, y_{i} \in Y, 1 \leqq i \leqq n$, and the infimum is taken over all finite chains $y=y_{0}, y_{1}, \cdots, y_{n}=z$.

REMARK. $(1) \Leftrightarrow(2)$ is the main theorem of $[1]$.

\section{REFERENCE}

1. C. J. Himmelberg, Preservation of pseudo-metrizability by quotient maps, Proc. Amer. Math. Soc. 17 (1966), 1378-1384.

UNIVERSITY OF KANSAS 